\title{
ESTRUTURA POPULACIONAL DE Pouteria macrophylla (Lam.) Eyma NA RESERVA EXTRATIVISTA TAPAJÓS-ARAPIUNS
}

\author{
Daniele Lima da Costa'; João Ricardo Vasconcellos Gama²; Misael Freitas dos Santos ${ }^{3}$; Renato Bezerra da \\ Silva Ribeiro ${ }^{4}$; Lia Oliveira Melo ${ }^{5}$; Oberdam Müller Moraes das Flores ${ }^{6}$; Hanna Kassia Machado da Silva ${ }^{7}$; \\ Girlene da Silva Cruz ${ }^{8}$.
1Universidade Federal do Oeste do Pará (UFOPA), Santarém, Pará, Brasil. danielelimadacosta@gmail.com
2UFOPA, Santarém, Pará, Brasil. jrvgama@gmail.com
3UFOPA, Santarém, Pará, Brasil. misael02freitas@gmail.com
4UFOPA, Santarém, Pará, Brasil. florestalrenatoribeiro@gmail.com
5UFOPA Santarém, Pará, Brasil. Icolivei@gmail.com
6UFOPA, Santarém, Pará, Brasil. muller30@gmail.com
7UFOPA, Santarém, Pará, Brasil.rhanna_ptr@hotmail.com
8UFOPA, Santarém, Pará, Brasil. girlene.lenecruz@gmail.com

RESUMO: O conhecimento da estrutura populacional de espécies com potencial de uso que não o madeireiro é relevante para destacar outros aproveitamentos da floresta, bem como, o estudo de seu potencial econômico permite elucidar a rentabilidade desse aproveitamento. Objetivou-se avaliar a estrutura populacional de Pouteria macrophylla na reserva extrativista Tapajós-Arapiuns e desse modo indicar se a espécie tem potencial para o comércio de seu fruto. Foram alocadas 202 parcelas (30 m x 250 m). Em cada parcela mensuraram-se todos os indivíduos com diâmetro à altura de $1,30 \mathrm{~m}$ do solo $\geq 10 \mathrm{~cm}$, considerando as seguintes classes de tamanho (CT) e tamanhos de parcelas: CT 1 $10 \mathrm{~cm} \leq$ DAP $<25 \mathrm{~cm}$ em subparcela de $30 \mathrm{~m} \times 50 \mathrm{~m} ;$ CT $2-25 \mathrm{~cm} \leq$ DAP $<50 \mathrm{~cm}$ em subparcela de $30 \mathrm{~m} \times 100 \mathrm{~m}$; e CT 3 - DAP $\geq 50 \mathrm{~m}$ na parcela de $30 \mathrm{~m} \times 250 \mathrm{~m}$. A espécie apresentou distribuição diamétrica na forma de $J$ invertido e distribuiu-se de modo agregado. O estudo indicou que a espécie Pouteria macrophylla tem potencial para comercialização de frutos e pode proporcionar uma renda complementar para comunitários.

PALAVRAS-CHAVE: Comercialização, Fitossociologia, Potencial de uso, Produto florestal não madeireiro.

\section{POPULATION STRUCTURE OF Pouteria macrophylla (Lam.) Eyma IN THE TAPAJÓS-ARAPIUNS EXTRACTIVE RESERVE}

ABSTRACT: The knowledge of the population structure of species with potential for uses other than the non-timber is relevant to highlight other uses of the forest, as well as, the study of its economic potential can elucidate the profitability of such use. The objective of this study was to evaluate the population structure and valuation of the fruit of Pouteria macrophylla in tapajós-arapiuns, and thus indicate that the species has potential in the area for the trade of its fruit. Were allocated 207 plots $(30 \mathrm{~m} \times 250 \mathrm{~m})$. And In each plot, were all individuals with diameter to the height of $1.30 \mathrm{~m}(\mathrm{DAP})=10 \mathrm{~cm}$ considering the following 
size classes (CT) and sizes of plots: CT $1-10 \mathrm{~cm} \leq$ DAP $<25 \mathrm{~cm}$ in subplot $30 \mathrm{~m} \times 50 \mathrm{~m}$; CT $2-25 \mathrm{~cm} \leq$ DAP $<50 \mathrm{~cm}$ in subplot $30 \mathrm{~m} \times 100 \mathrm{~m}$; and CT 3 - DAP $=50 \mathrm{~m}$ in subplot 30 $\mathrm{m} \times 250 \mathrm{~m}$. The species presented diametric distribution in the form of inverted J and distributed in such a way as aggregate. The valuation of standing forest indicated that Pouteria macrophylla has potential for commercialization of fruits and can provide an income supplement for community.

KEYWORDS: Marketing, Non- timber forest product, Phytosociology, Potential use.

\section{ESTRUCTURA POBLACIONAL DE Pouteria macrophylla (Lam.) Eyma EN LA RESERVA EXTRACTIVA TAPAJÓS-ARAPIUNS}

RESUMEN: El conocimiento de la estructura poblacional de especies con potencial de uso que no sea el maderero es relevante para destacar otros aprovechamientos del bosque, así como el estudio de su potencial económico permite elucidar la rentabilidad de ese aprovechamiento. Se objetivó evaluar la estructura poblacional y valoración del fruto de Pouteria macrophylla en la reserva extractivista Tapajós-Arapiuns, y de ese modo indicar si la especie tiene potencial en el área para el comercio de su fruto. Se asignaron 207 parcelas (30 m x 250 m). En cada parcela, se midieron todos los individuos con diámetro a la altura de 1,30m (DAP) $\geq 10 \mathrm{~cm}$ considerando las siguientes clases de tamaño (CT) y tamaños de parcelas: CT $1-10 \mathrm{~cm} \leq$ DAP $<25 \mathrm{~cm}$ en subparcela de $30 \mathrm{~m} \times 50 \mathrm{~m}$; CT $2-25 \mathrm{~cm} \leq$ DAP $<50 \mathrm{~cm}$ en subparcela de $30 \mathrm{~m} \times 100 \mathrm{~m}$; y CT 3 - DAP $\geq 50 \mathrm{~m}$ en la parcela de $30 \mathrm{~m} \times 250$ m. La especie presentó distribución diamétrica en la forma de J invertido y se distribuyó de modo agregado. La valoración del bosque en pie indicó que Pouteria macrophylla tiene potencial para comercialización de frutos y puede proporcionar una renta complementaria para comunitarios.

PALABRAS CLAVE: Comercialización, Fitosociología, Potencial de uso, Producto forestal no maderero.

Muitas espécies frutíferas ocorrentes da região amazônica têm grande importância para as populações tradicionais, sendo fonte de alimento e usadas para obtenção de renda. Buscar informações sobre a estrutura populacional dessas espécies é fundamental para entender seus processos dinâmicos e ecológicos, que são fundamentais para execução de manejo, bem como para avaliar o potencial de uso de determinada espécie.

Há muito tempo as florestas vêm sendo valorizadas pela diversidade de produtos que delas provém, são inúmeros seus 
benefícios e potencialidades. Os produtos florestais não madeiráveis são oriundos principalmente das florestas nativas, sendo a floresta amazônica a principal fonte de fornecimento destes produtos, que incluem: frutas, castanhas, resinas, látex, óleos essenciais, fibras, forragem, fungos, fauna e madeira para a fabricação de artesanato (SOUZA, 2011).

A Amazônia abrange uma grande diversidade de árvores frutíferas, dentre as espécies frutíferas encontra- se o abieiro (Pouteria macrophylla (Lam.) Eyma), é uma planta tropical, originária da região amazônica, encontrada desde as encostas andinas do Peru, se estende até a parte oeste da amazônia brasileira, adaptou-se facilmente para cultivo nas regiões litorâneas do Oiapoque (AM) até Santos (SP), hoje também encontrada em regiões do interior do país onde apresentam solos profundos e humosos (SEGALLIO, 2009).

○ gênero Pouteria pertencente à família Sapotaceae é encontrado na região amazônica na mata pluvial e, na sua transição para o cerrado e campina (LOZENZI, 2009), possui espécies utilizadas na alimentação, em construções civis e na medicina popular (SILVA, 2009). O fruto do abieiro é uma baga ovóide ou esférica, solitária, com exocarpo amarelado quando maduro, que exuda um látex (SEGALLIO, 2009).

$\bigcirc$ fruto abiu é muito popular, com polpa comestível, possui consistência gelatinosa, branca ou amarelada, doce, e contém de 1 a 4 sementes, lisas, brilhantes, pretas, e de tamanho variável (SEGALLIO, 2009). Sua polpa é bastante utilizada na produção de sorvetes, cremes, doces e salgados (MINISTÉRIO DA SAÚDE, 2015).

Nesse sentido, há necessidade de conhecimento da composição florística, estrutura e distribuição espacial das espécies vegetais, visando com isso planificar o manejo para produção de diversos produtos madeireiros e não madeireiros comercializáveis (CARIM, 2007). Desse modo, o objetivo do presente estudo foi avaliar a estrutura populacional de Pouteria macrophylla na Reserva Extrativista Tapajós-Arapiuns, e com base nessas informações indicar se a espécie tem potencial na área para o comércio de seu fruto.

A área de estudo localiza-se na Área de Manejo Florestal (AMF) da Reserva Extrativista (RESEX) Tapajós-Arapiuns, que 
está situada às margens dos Rios Tapajós e Arapiuns, fazendo parte dos Municípios de Aveiro e Santarém. Abrange aproximadamente 62.000 ha, com cerca de 18.000 moradores que vivem em mais de 70 comunidades.

O clima da região, pela classificação de Koppen, é do tipo Ami, com temperatura média anual de $25,5^{\circ} \mathrm{C}$. A concentração de chuvas ocorre entre janeiro e maio, resultando em uma precipitação média anual de $1.820 \mathrm{~mm}$. O solo é do tipo Latossolo Amarelo Distrófico. A vegetação é classificada como Floresta Ombrófila Densa, caracterizando-se pela dominância de indivíduos arbóreos de grande porte e pela abundância de lianas lenhosas, palmeiras e epífitas (IBGE, 2012).

Foram alocadas, sistematicamente, 202 parcelas de área fixa de 30 m x 250 m em faixas distantes $4 \mathrm{~km}$ uma da outra, totalizando uma amostragem de 155,25 ha. Dentro de cada faixa a distância entre as parcelas foi de 500 m. Em cada parcela inventariou-se as seguintes classes de tamanho (CT): CT1 - $10 \mathrm{~cm} \leq \mathrm{DAP}<25 \mathrm{~cm}$ em subparcela de 30 m x 50 m; CT2 - 25 $\mathrm{cm} \leq \mathrm{DAP}<50 \mathrm{~cm}$ em subparcela de 30 m x 100 m; e CT3 - DAP $\geq 50$ cm na parcela de $30 \mathrm{~m} \times 250 \mathrm{~m}$.

Os parâmetros fitossociológicos de abundância e dominância foram estimados conforme Souza e Soares (2013). A estrutura dos indivíduos foi obtida a partir da distribuição da densidade destes, por centros de classes diamétricas com amplitude de $10 \mathrm{~cm}$ (RIBEIRO, 2013).

Para verificar a forma como a população se distribuía espacialmente, utilizou-se o índice de Morisita, conforme recomendação de Brower e Zar (1977), por ser de grande utilidade na conservação e no manejo florestal, pois a sua interpretação produz subsídios importantes para a elaboração de modelos de restauração de áreas degradadas, enriquecimentos florestais e escolhas de técnicas aplicadas ao manejo florestal (FREITAS; MAGALHÃES, 2014; BENTESGAMA et al. 2002):

$$
I M_{i}=\frac{n \cdot\left(\Sigma x^{2}-N\right)}{n(N-1)}
$$

Em que: IM = Índice de Morisita da i-ésima espécie; $\mathrm{n} \mathrm{i}=$ número total de parcelas amostradas; $\mathrm{N}=$ número total de indivíduos, contidos nas $n$ parcelas; $X^{2}=$ quadrado do número de indivíduos por parcela. 
Se $I M=1,0$, infere-se que a i-ésima espécie tem padrão de distribuição espacial aleatório; se IMi < 1,0, o padrão de distribuição espacial da i-ésima espécie é uniforme ou regular; e se $\mathrm{IMi}>1,0$, o padrão de distribuição é agregado. O nível de significância dos valores de IM foi obtido pelo teste Qui-quadrado:

$$
X^{2}=\frac{n \cdot \Sigma x^{2}}{N}-N
$$

Em que: $X^{2}$ : valor de qui-quadrado; e $n, N, s, X^{2}$ : já definidos anteriormente.

A interpretação do valor de quiquadrado baseou-se no seguinte: se o valor calculado for menor que o valor tabelado o IM não difere significativamente de 1 e a espécie apresentará um padrão de distribuição aleatória. Porém, se o valor qui-quadrado for maior que o tabelado a espécie tenderá a um padrão de distribuição agregada.

A produtividade de frutos de Pouteria macrophylla foi estimada utilizando seguinte equação:

$$
\mathrm{PM}_{\mathrm{i}}=\mathrm{PS}_{\mathrm{i}} \cdot \mathrm{NA}_{\mathrm{i}}
$$

Em que $\mathrm{PM}_{\mathrm{i}}=$ Produtividade média anual da $i$ ésima espécie em kg.ha-1 ${ }^{1}$ PS ${ }_{i}=$ Produção média anual de frutos da $i$ ésima espécie em kg; $N A_{i}=$ número de árvores da i ésima espécie (DAP $\geq 20 \mathrm{~cm}$ ) em arv.ha-1.
A espécie Pouteria macrophylla apresentou densidade de 2,93 arv.ha-1 e dominância em área basal de 0,1624 $\mathrm{m}^{2} \cdot \mathrm{ha}^{-1}$. Em floresta de terra firme no estado do Pará, Almeida et al. (2012) encontrou densidade superior a deste estudo para mesma espécie, 14,75 árv.ha-1, para indivíduos com DAP $\geq 10 \mathrm{~cm}$. Reis et al. (2013) também encontrou densidade superior, 9 árv.ha-1 com DAP de inclusão $\geq$ 10 cm, no município de Moju também no Pará.

A distribuição de densidade das árvores por classe de tamanho (CT), demonstra forma assimétrica positiva, com 66,52\% das árvores ocorrendo na CT 1, 31\% pertencentes a CT 2 e 3,48\% apresentadas na CT3 (Figura 1A). Esta forma de distribuição pode ser considerada um fator importante para permanência da espécie na área, uma vez que os indivíduos regenerantes apresentam maior densidade, indicando que há manutenção do equilíbrio da espécie.

A distribuição das árvores por centro de classes diamétricas apresentou a característica forma de J invertido (Figura 1B), que é a forma comumente esperado para distribuições diamétricas de florestas 
nativas (CABACINHA e CASTRO, 2010; ALVES JÚNIOR et al., 2007). Gomide (2009), também encontrou grande densidade de árvores nas menores classes de diâmetros e infere que desse modo é possível que as espécies forneçam parte de seus representantes para as classes subsequentes, durante os períodos futuros, auxiliando na dinâmica e garantindo a continuidade arbórea da floresta. Isso se deve ao potencial que as espécies vegetais têm em se regenerar (LIMA, 2013).

Figura 1. Distribuição das classes de tamanho (A) e diamétrica (B) de Pouteria macrophylla na área de manejo florestal madeireiro na RESEX Tapajós-Arapiuns.

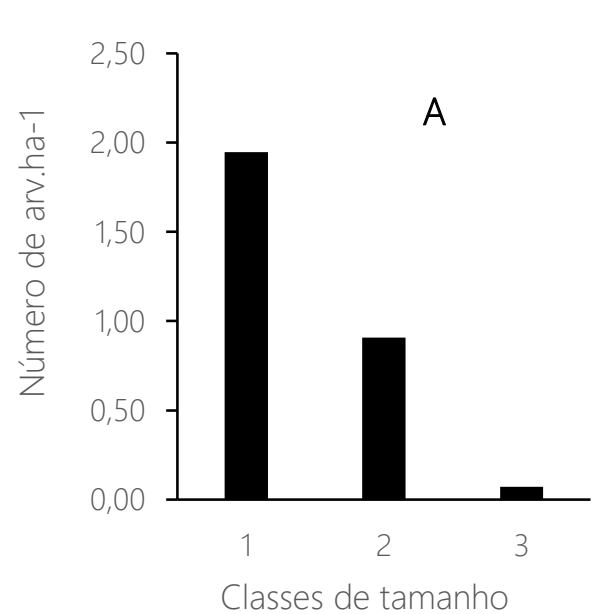

Essa análise demonstra que a população de $P$. macrophylla apresenta recrutamento de regeneração natural, o que garante a presença de árvores no estrato adulto desta espécie (VIEIRA et al., 2014). De acordo com esse modo de distribuição nas diferentes classes diamétricas observa-se que a população está em equilíbrio entre as classes.

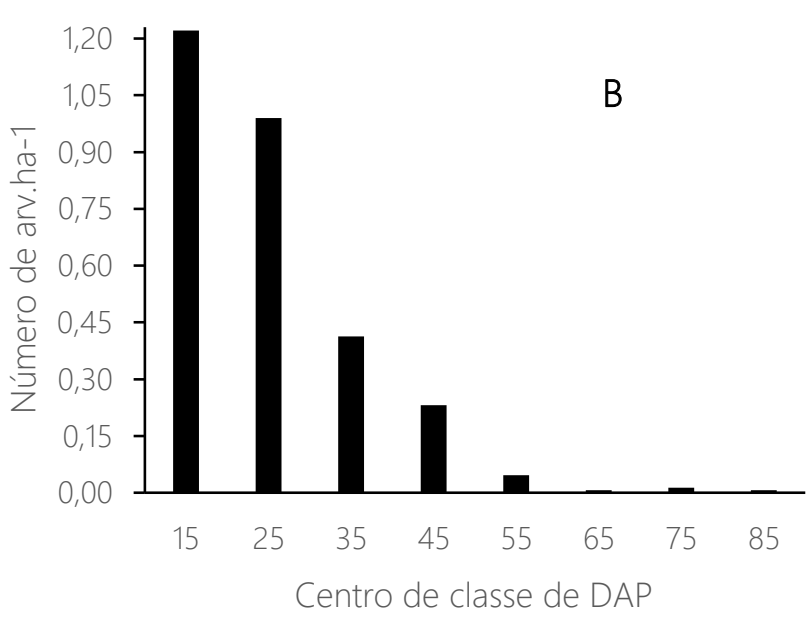

A avaliação da dispersão espacial da população por meio de Morisita, mostrou que esta, distribui-se de forma agregada, sendo confirmada essa agregação através do teste Qui-quadrado com significância $(p<0,05)$. A espécie pode apresentar este padrão devido ter encontrado condições ambientais que favorecem seu estabelecimento na área, e assim, se 
sobressai na competição para sua sobrevivência.

O padrão de distribuição agregado é comum nas florestas tropicais, sendo fator predominante, sugerindo que acha no ambiente uma força de atração, onde a chance de ocorrência de um indivíduo é aumentada pela presença de outros do mesmo, ou também pode ocorrer em função da disponibilidade de um determinado recurso comum (SILVA et al., 2008).

Se faz importante conhecer o padrão de distribuição das espécies para tomada de decisão no que se refere ao seu manejo, seja ele de interesse madeireiro ou não madeireiro. Sendo esse conhecimento fundamental para escolha dos métodos silviculturais a serem aplicados na área de manejo (FREITAS; MAGALHÃES, 2014).

Para espécies utilizadas para comercialização madeireira e de produtos não madeireiros, que apresentam padrão de distribuição agregado, devem-se manter alguns indivíduos de tamanho comercial, para que continue a ocorrer a distribuição natural (VIEIRA, 2014).

Considerando as árvores com DAP $\geq 20$ cm, e produção de frutos média anual por árvore de 77 kg, de acordo com Falcão (1999). A produtividade média anual de frutos de P. macrophylla foi estimada em $225.61 \mathrm{~kg} \cdot \mathrm{ha}^{-1}$. Vale ressaltar que a produção de frutos varia de planta para planta, depende do ambiente em que o indivíduo se localiza, disponibilidade de nutrientes, fitossanidade da árvore e demais fatores que influenciam no tamanho e peso do fruto (FALCÃO, 1999).

Conforme Lorenzi (2009) os frutos de $P$. macrophylla são bastante apreciados pela população do norte do país e oferecidos em feiras livres, sendo regionalmente cultivada em pomares domésticos. Falcão, (1999) já discutia a importância do gênero Pouteria no comércio de frutas na região norte, relatando que este fruto desperta não somente o interesse regional, como também a nível nacional e internacional.

Segundo Guerra (2008) a extração de produtos florestais não madeireiros tem importante papel na economia das comunidades tradicionais, que além de uma atividade de subsistência, é fonte de renda para estas comunidades. Observase que P. macrophylla é promissora para a comercialização de frutos pelas comunidades que estão próximas a área 
estudada, garantindo fonte de renda complementar, além de atribuir usos a espécie que não seja apenas seu aproveitamento madeireiro.

A espécie apresentou estoque de regeneração natural, estoque de crescimento e padrão de dispersão agregado, fatores que podem garantir a continuidade da produção de frutos e facilita a coleta dos mesmos. Desse modo, a mesma tem potencial para comercialização de seu fruto, podendo gerar benefícios econômicos às comunidades.

\section{REFERÊNCIAS}

ALMEIDA, L. S.; GAMA, J. R. V.; OLIVEIRA, F.A.; CARVALHO, J. O. P.; GONÇALVES, D. C. M.; ARAÚJO, G. C. Fitossociologia e uso múltiplo de espécies arbóreas em floresta manejada, Comunidade Santo Antônio, município de Santarém, Estado do Pará. Acta Amazonica, v.42, n.2, p.185 - 194, 2012.

ALVES JÚNIOR, F. T.; LINS, C. F.; BRANDÃO, S.; ROCHA, K. D.; SILVA, J. T.; MARAGON, L. C. ET AL. Estrutura diamétrica e hipsométrica do componente arbóreo de um fragmento de mata atlântica, Recife-PE. Cerne; v.13, n.1, p.8395, 2007.

BENTES-GAMA, M. M.; SCOLFORO, J. R. S.; GAMA, J. R. V.; OLIVEIRA, A. D. Estrutura e Valoração de uma Floresta de Várzea Alta na Amazônia. Revista Cerne, v.8, n.1, p.88102, 2002.

BROWER, J. E.; ZAR, J. H. Field and laboratory methods for general ecology. 2. ed. Dubique: Win. C. Brown Publishers, 1977. 226 p.

CABACINHA, C. D.; CASTRO, S. S. Estrutura Diamétrica e Estado de Conservação de Fragmentos Florestais no Cerrado Brasileiro. Floresta e Ambiente, v.17, n.1, p.51-62, 2010.

CARIM, S.; SCHWARTZ, G.; SILVA, M. F. F. Riqueza de espécies, estrutura e composição florística de uma floresta secundária de 40 anos no leste da Amazônia. Acta bot. bras., v.21, n.2, p.293308, 2007.

CALÉGARIO, N.; SOUZA, A. L.; MARANGON, L. C.; SILVA, A. F. Estimativa dos parâmetros de distribuição e de associação de espécies vegetais nativas regeneradas no sub-bosque Eucalyptus, no Município de Belo Oriente, MG. Revista Árvore, v.7, n.2, p.146-161, 1993.

FALCÃO, M. A.; CLEMENT, C. R. Fenologia e produtividade do abiu (Pouteria caimito) na Amazônia Central. Acta Amazônica v.29, p.3-11,1999.

FREITAS, W. K., MAGALHÃES, L. M. S. Florística, diversidade e distribuição espacial das espécies arbóreas de um trecho de floresta estacional semidecidual da serra da concórdia, RJ. Floresta, v.44, n.2, p.259 - 270, 2014.

GOMIDE, L. R.; SCOLFORO, J. R. S.; OLIVEIRA, A. D. Análise das estruturas diamétrica e hipsométrica de fragmentos florestais localizados na bacia do rio São 
Francisco, em Minas Gerais, Brasil. Floresta, v.39, n.2, p.239-251, 2009

GUERRA, F. G. P.Q. Contribuição dos produtos florestais não madeireiros na geração de renda na Floresta Nacional do Tapajós - Pará. Curitiba, 2008. 119 f. Dissertação (Mestrado em Ciências Florestais). Universidade Federal do Paraná, Curitiba. 2008.

IBGE. Manual Técnico da Vegetação Brasileira, Rio de Janeiro: IBGE, 2012.

LIMA, J. P. C.; LEÃO, J. R. A. Dinâmica de Crescimento e Distribuição Diamétrica de Fragmentos de Florestas Nativa e Plantada na Amazônia Sul Ocidental. Revista Floresta e Ambiente. v.20, n.1, p.70-79, 2013.

LORENZI, H. Árvores brasileiras: manual de identificação e cultivo de plantas arbóreas nativas do Brasil. Nova Odessa: Instituto Plantarum, v.2, ed.3, 384 p., 2009.

MINISTÉRIO DA SAÚDE, Secretaria de Atenção à Saúde Departamento de Atenção Básica. Alimentos Regionais Brasileiros, 2 ed., 486 p., Brasília - DF, 2015.

PAES-DE-SOUZA, M.; SILVA, T. N.; PEDROZO, E. A.; FILHO, T. A.S. O Produto Florestal Não Madeirável (PFNM) Amazônico açaí nativo: proposição de uma organização social baseada na lógica de cadeia e rede para potencializar a exploração local. Revista de Administração e Negócios da Amazônia, v.3, n.2, p.44-57, 2011.

REIS, L. P; SILVA, J. N. M.; REIS, P. C. M.; CARVALHO, J. O. P:; QUEIROZ, W. T.; RUSCHEL, A. R. Efeito da exploração de impacto reduzido em algumas espécies de
Sapotaceae no leste da Amazônia. Floresta, v.43, n.3, p.395-406, 2013.

RIBEIRO, R. B. S.; GAMA, J. R. V.; MARTINS, S. V.; MORAES, A.; SANTOS, C. A. A.; CARVALHO, A. D. Estrutura florestal em projeto de assentamento, comunidade São Mateus, município de Placas, Pará, Brasil. Revista Ceres, Viçosa, v.60, n.5, p.610 - 620, 2013.

SEGALLIO, R. M. Estudo de extração do látex e do óleo essencial do abiu, 2009, in: Congresso de Iniciação Científica e $7^{\circ}$ amostra acadêmica UNIMEP, Piracicaba. Anais do $17^{\circ}$ Congresso de Iniciação Científica e $7^{\circ}$ amostra acadêmica UNIMEP, 2009.

SILVA, M. A.; MELLO, J. M.; SCOLFORO, J. R. S.; CZANCK JÚNIOR, L.; ANDRADE, I. S.; OLIVEIRA, A. D. Análise da distribuição espacial da candeia (Eremanthus erythropappus (DC.) MacLeish) sujeita ao sistema de manejo porta-sementes. Cerne, v.14, n.4, p.311 - 316, 2008.

SOUZA, A. L.; SOARES, C. P. B. Florestas nativas: estrutura, dinâmica e manejo. Viçosa, MG: Editora UFV, 1. ed. 2013, 322 p.SILVA, C. A. M.; SIMEONI, L. A.; SILVEIRA, D. Genus Pouteria: chemistry and biological activity. Revista brasileira de farmacognosia. v.19 n.2. 2009.

VIEIRA, D. S.; GAMA, J. R. V.; ANDRADE, D. F. C. Estrutura populacional e padrão de distribuição espacial de Pouteria cladantha Sandwith em uma floresta sob regime de manejo sustentável, Pará. Biota Amazonia, v.4, n.3, p.42-47, 2014. 